\title{
Signal Processing for AUV based Interferometric Synthetic Aperture Sonar
}

\author{
Roy Edgar Hansen, Torstein Olsmo Sæbø \\ and Kenneth Gade \\ Norwegian Defence Research Establishment (FFI) \\ P O Box 25, NO-2027 Kjeller, Norway \\ Roy-Edgar.Hansen@ffi.no, Torstein-Olsmo.Sabo@ffi.no \\ Kenneth.Gade@ffi.no
}

\author{
Sean Chapman \\ QinetiQ, Bincleaves \\ Newton Road Weymouth, Dorset DT4 8UR, UK \\ sachapman@qinetiq.com
}

\begin{abstract}
This paper presents signal processing techniques particularly suited for interferometric Synthetic Aperture Sonar (SAS) systems onboard Autonomous Underwater Vehicles (AUV) (or other platforms carrying high grade navigation systems). The signal processing is applied to data collected in a controlled rail experiment at Elba Island, Italy, using a wideband interferometric SAS and an Inertial Navigation System (INS).

We evaluate different strategies in fusing sonar micronavigation by the Displaced Phase Center Antenna (DPCA) technique with Aided INS (AINS). We obtain highest navigation accuracy using DPCA as aiding sensor into the AINS, then using raw DPCA surge and sway in combination with the AINS attitude and position.

Coarse cross correlation based bathymetry and full resolution interferometry (based on the interferogram) is tested on the full swath and objects. Coarse bathymetry is more reliable than the interferogram technique. Phase wraparounds are avoided by estimating the coarse bathymetry first, then using the full resolution phase estimates as correction. Although much work remains, this technique does show a clear potential in improving object classification ability.
\end{abstract}

\section{INTRODUCTION}

The Synthetic Aperture Sonar (SAS) principle is based on increasing the sonar image azimuth resolution by coherent combination of data from succesive pings. The technique has the potential to improve the azimuth resolution by one order of magnitude or more compared to conventional Side Scan Sonars (SSS). SAS requires a stable slow moving platform, and SAS processing gives better results with other navigation sensors available. This makes SAS the ideal primary sensor onboard Autonomous Underwater Vehicles (AUV) performing seafloor imaging. In military applications, the SSS to SAS increase in resolution facilitates object classification at detection ranges.

The Norwegian authors work in a joint project between the Norwegian Defence Research Establishment (FFI) and Kongsberg Simrad to develop a prototype interferometric SAS for the HUGIN AUV [1]. The project is part of the Norwegian military AUV program [2] to deliver a prototype AUV to the Royal Norwegian Navy (RNoN) for installation on the Oksøy class mine hunters (see Fig 1).

FFI and QinetiQ participate in the Joint Reseach Program Mine Detection and Classification at SACLANTCEN. In november 2000, a series of trials named InSAS-2000 were conducted at Elba Island (Italy) with SACLANTCEN, Qinetiq and FFI as participants [3]. The basis of the experiment was to perform controlled motion of a scaled interferometric SAS onboard a trolley with a high grade Inertial Navigation System (INS). The experimental results in this paper are based on the InSAS-2000 trials.

\section{SAS Signal Processing Overview}

An overview of an interferometric synthetic aperture sonar mounted on an AUV is shown in Fig 2. The sonar consists of a phased array transmitter and two along-track receiver arrays. The length of the receiver arrays determines the area coverage rate, while the size of each element (or number of elements) determines the theoretical azimuth resolution [4], [1]. Two receiver arrays are vertically displaced, giving the ability to perform interferometric processing for estimation of bathymetry. The slant-range plane is defined as the plane for which the acoustic waves are within for a given sonar location and specified range. When the AUV moves along the path, all pings are collected and stored. The data can then either be processed conventionally as a dynamically focused side scan sonar, or synthetic aperture processing can be applied.

The SAS signal processing chain shown in Fig 3 can be divided into four different parts:

\section{A. Motion Estimation}

Motion estimation, or navigation, constitutes estimation of platform motion, either from the sonar data, or an INS, or a combination of the two. Sonar micronavigation is performed

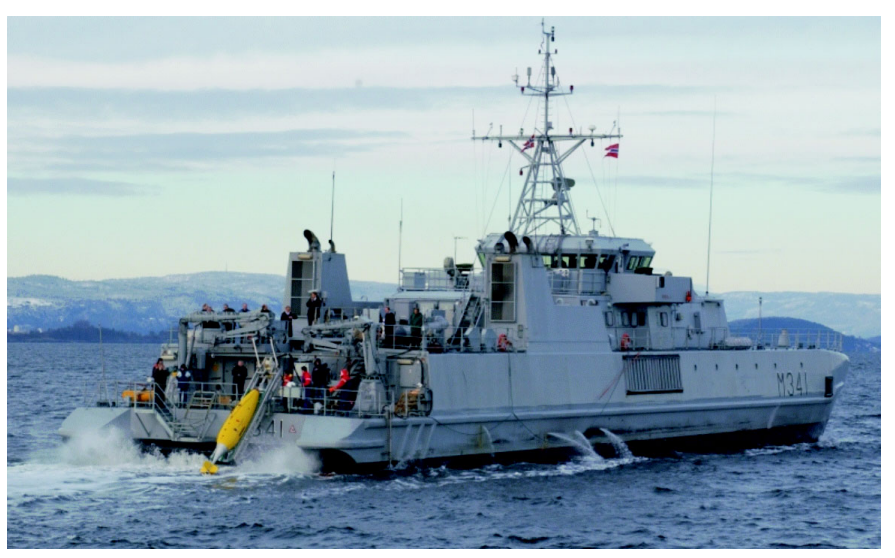

Fig. 1. The HUGIN AUV immediately before lauch from the RNoN mine hunter KNM Karmøy in December 2001. 


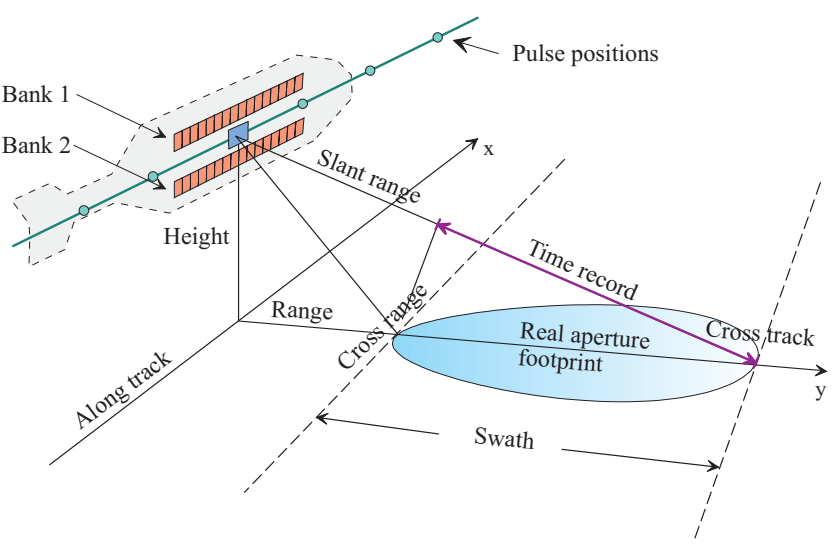

Fig. 2. Overview of an interferometric SAS system on an AUV.

by the Displaced Phace Center Antenna (DPCA) technique [5], [6], [7]. The principle of DPCA is to estimate alongtrack displacement (surge), cross-track displacement (sway) and cross-track rotation (yaw) based on ping to ping cross correlations of overlapping transmitter-receiver pairs. This technique requires redundant sonar data by running at lower AUV speed than required by the synthetic aperture spatial sampling criterion. Hence, the coverage rate is reduced by using DPCA micronavigation. The limiting factor for DPCA alone for motion estimation is accumulated yaw error [7].

The HUGIN AUV does carry a high grade Aided Inertial Navigation System (AINS) providing attitude orders of magnitude more accurate than DPCA yaw. The theoretical accuracy of DPCA surge and sway far exceeds the accuracy of the AINS. This makes the combination of these two navigation techniques an attractive solution for SAS imagery [3]. Fusion of DPCA micronavigation and inertial navigation also has the potential to improve the AUV navigation autonomy [8]. Integrating DPCA motion estimation with INS does, however, require estimation of the depression angle in order to couple the acoustic motion estimation into the INS. Hence, swath bathymetry ping by ping (broadside interferometry) is required within the motion estimation.

\section{B. Beamforming}

Beamforming by synthetic aperture processing can be done in two classes of ways. Within the straight line assumption, the wavenumber [9], [10] and chirp-scaling algorithm [11] can be used. This requires, however, that motion compensation is performed before synthetic aperture imaging. These techniques are fast, but have limitations to the degree of deviation from straight line [12], [13]. The other class of beamforming tolerates arbitrary motion and does not require any motion compensation. Time domain beamforming [14] is orders of magnitude slower than all other listed techniques. This is also the only technique that does not compromise any image quality for speed. Fast factorized backprojection [15], [16] have the same computational load as the wavenumber algorithm, but on the cost of image SNR [17].

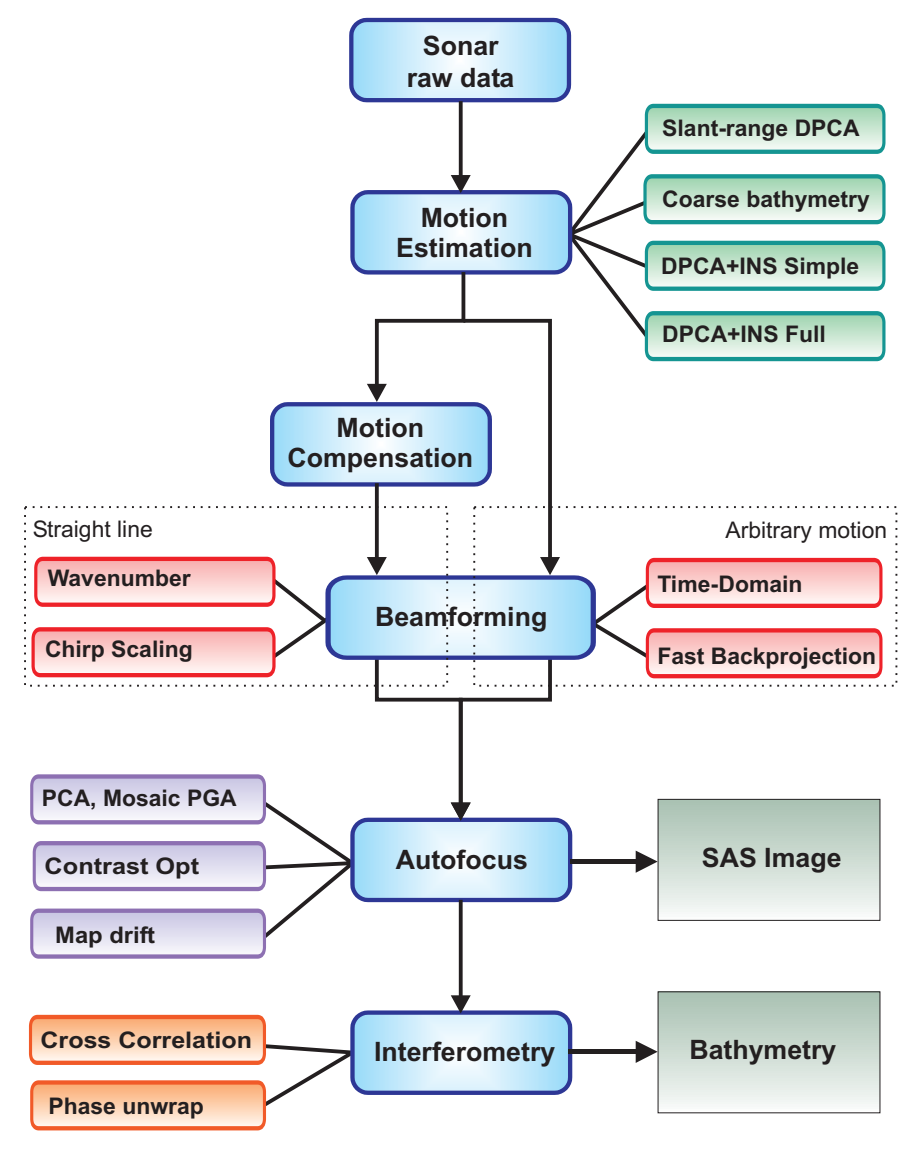

Fig. 3. SAS Signal processing overview.

\section{Autofocus}

Autofocus are postprocessing techniques to correct for uncompensated motion errors or medium irregularities. Most of these techniques are adapted directly from the SAR community, and does not perform optimally on SAS images without modification. This is due to the fact that SAS systems are wideband and broadbeam compared to typical SAR systems [18]. The Phase Gradient Algorithm (PGA) family of techniques [12], [19] show promising results in SAS autofocusing.

\section{Interferometry}

Interferometric processing constitutes estimation of bathymetry from vertical displaced receivers. There are two classes of techniques: Cross correlation techniques [20], [21] produce bathymetric maps at reduced resolution. Interferometry by two-dimensional phase unwrapping [11], [22] has the potential to produce full resolution bathymetric maps. Full resolution SAS interferometry with 2D phase unwrapping is non-trivial and not considered as generally solved as of today.

\section{EXPERIMENTAL RESULTS FROM INSAS-2000}

The sonar (right image) and the rail (left image) are shown in Fig 4. The sonar consists of two along-track receiver arrays and three displaced transmitters mounted on a trolley. A high grade Inertial Measurement Unit (IMU) is also placed on the 

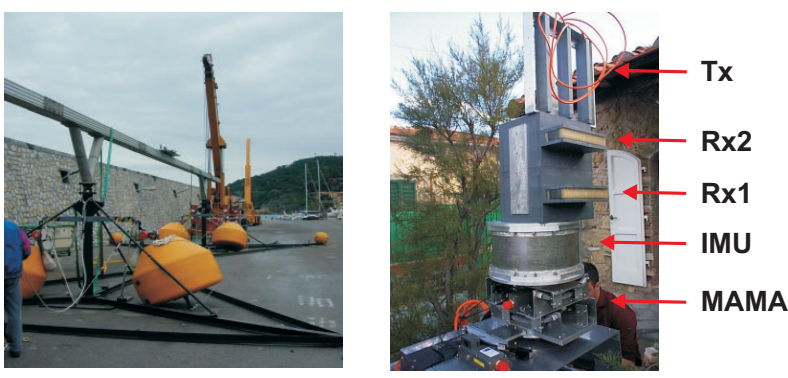

Fig. 4. Left: Rail. Right: Sonar with IMU and MAMA.

trolley, A Multi Axis Motion Actuator (MAMA) was used to force different types of motion (mechanical yaw, roll and sway in addition to surge along the rail). See [3] for details regarding the experiment.

Figure 5 shows a SAS image of run 221113 from InSAS2000. The synthetic aperture image is produced by coherent summation of 500 pings, equivalent to $16.7 \mathrm{~m}$ (full length of the rail). The sonar is run with overlap factor 4 (4 times slower than required by the spatial sampling criterion for synthetic aperture imagery). This gives a theoretical SAS to sidescan sonar azimuth resolution improvement (or Q-factor) of 125.75 [7]. This SAS image is produced by full integration of DPCA and INS (see the next section), dynamic focusing by time domain interpolation beamforming [14] and no autofocusing. The image contains 5 different targets: a rock (lower left), a truncated cone (lower center), a triangular shape (lower right), a bicycle (upper left) and a ladder (upper right).

The challenge in seafloor imaging in military applications, is to produce images of such quality that man-made objects can be separated from natural objects (stones) of equal size with highest possible presision (not missing any and not having too many false alarms). In this particular case, the two geometrical

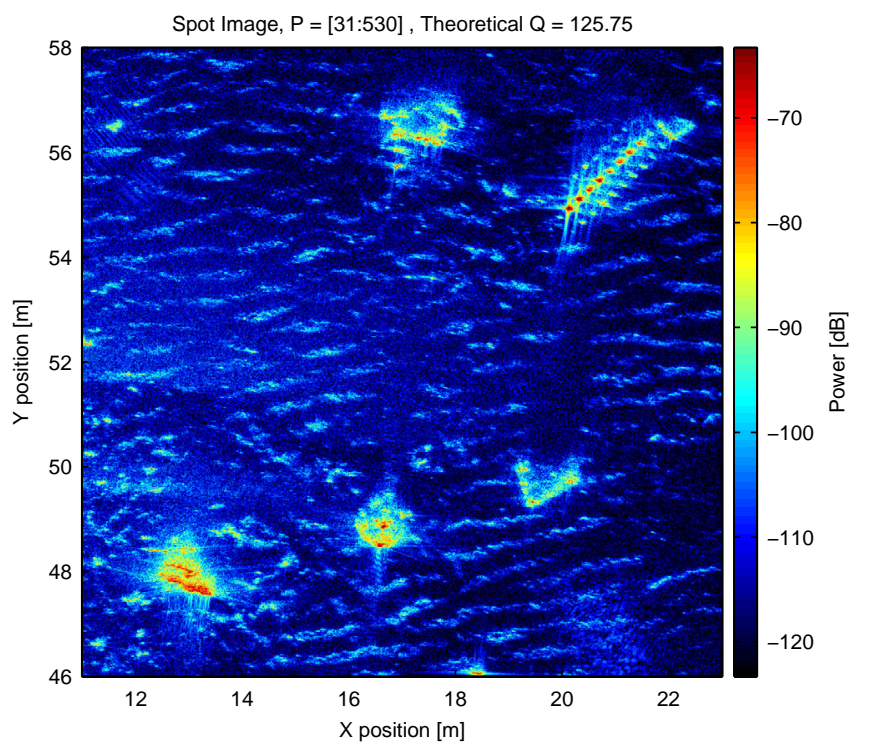

Fig. 5. SAS image of the target field from InSAS-2000.
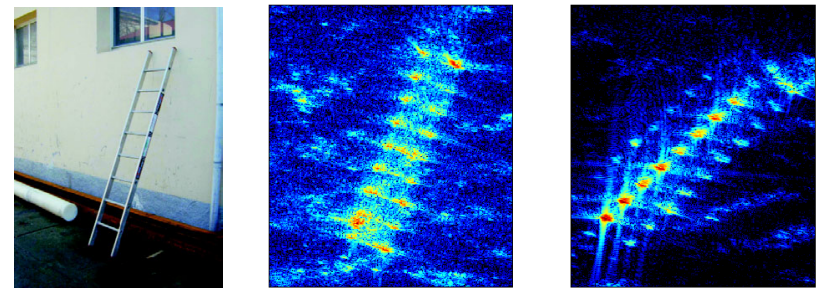

Fig. 6. Optical image (left) and SAS images from run 221107 (center) and run 221113 (right) of a $2.1 \mathrm{~m}$ ladder with 8 steps.

shapes on the seafloor are mine-like objects, and the stone is of similar size. Classification of objects in mine hunting is then to determine of which certainty each of the detected objects are mines.

Figure 6 shows the SAS image of the ladder from run 221113 and run 221107. The object is at different locations and with different orientation at the two runs. Both images show 8 steps on the ladder. In addition we see the start (or end) of the ladder on the center image (as a ninth step). The $\mathrm{V}$-shape at the upper end of the right-most SAS image is not part of the ladder. Recognizing the ladder in the SAS images is straight forward.

In Fig 7 we see the SAS images of the bicycle from the two runs above. Again, the bicycle is relocated between the two runs, and the handlebars and pedals are in different positions. Classifying the bicycle directly from the SAS images is clearly non-trivial. These two examples show that although "superclassification" can be performed on images approaching theoretical resolution of $1.5 \times 1.5 \mathrm{~cm}$, identification of objects still is best performed with optical sensors.
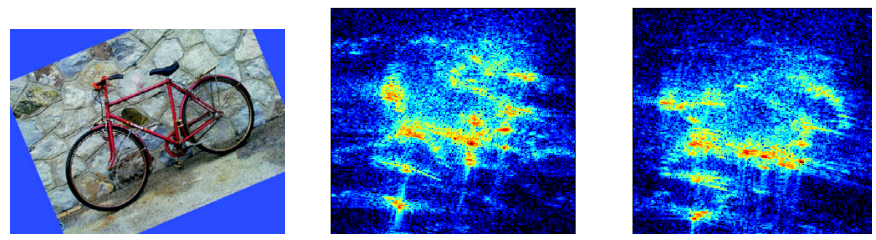

Fig. 7. Optical image (left) and SAS images from run 221107 (center) and run 221113 (right) of a bicycle.

\section{Navigation Details}

Accurate navigation is crucial for optimal use of synthetic aperture sonar. A maximum phase-error of $\pi / 4$ along the synthetic aperture leads to a position accuracy of $\lambda / 16$, equvialent to $1 \mathrm{~mm}$ for InSAS-2000. This accuracy is unrealistic for AUV motion along a synthetic aperture of $10-100 \mathrm{~m}$ with traditional navigation techniques.

There are usually measurements from three different sets of navigation sensors available: the IMU, DPCA and other aiding sensors such as a Doppler Velocity Logger (DVL). Figure 8 shows possible ways to combine these sensors. If switch 1 and 2 are both open, only IMU measurements and aiding sensors are used and evaluated in an error-state Kalman filter (INS). 


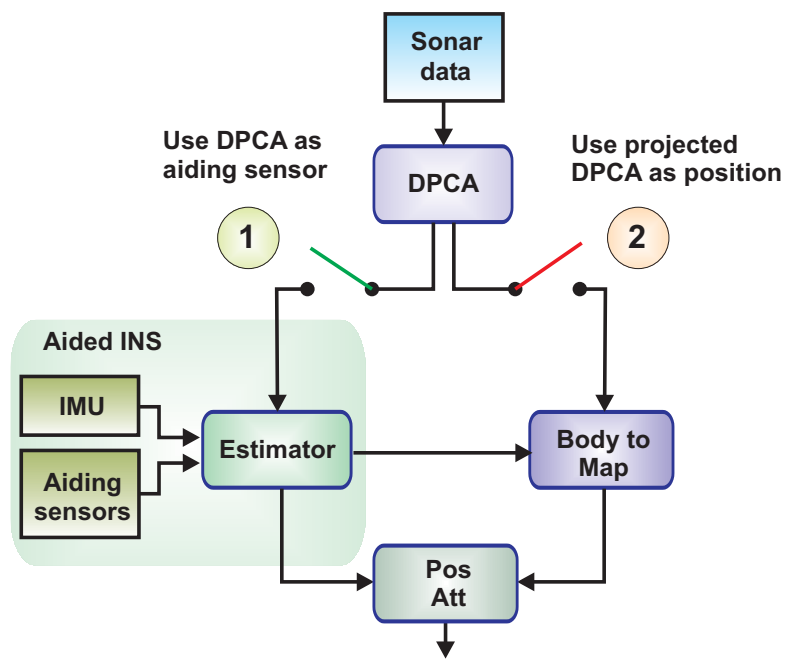

Fig. 8. Integration of DPCA micronavigation into the Aided INS.

DPCA can be treated as (a highly accurate) delta sensor. Closing switch number 1 adds DPCA as aiding sensor into the Kalman filter (DPCA-AINS). This integration requires the knowledge of the direction of the acoustic axis relative to the AUV (or slant-range direction, see Fig 2) for each ping, which can be obtained by interferometry. Alternatively, we can close switch 2 and use full integration of INS and DPCA. This technique consists of rotating the INS measurements into body coordinates, replacing the calculated surge and sway with DPCA measurements, and rotate the fused data back into map coordinates (INS \& DPCA). Also this integration technique requires ping by ping slant-range direction. Finally, it is possible to use both integrations simultaneously (DPCAAINS \& DPCA). This is the method we have used in this paper, unless specifically stated otherwise.

We have tested the four methods described above together with pure DPCA micronavigation and straight line assumption on run 221107 from InSAS-2000. The SAS images based on these 6 navigation strategies are shown in Fig 9. The images are produced with resolution $1 \times 1 \mathrm{~cm}$ based on 500 pings.

The visible effect of using DPCA as aiding sensor is marginal when also using full integration of DPCA and INS. Hence, DPCA surge and sway as aiding sensors have little impact on INS attitude. This is probably due to the short synthetic aperture (limited by the rail) and the short range in this particular experiment. In typical scenarios for AUV based SAS, DPCA aided INS have the potential to improve both the synthetic aperture length and the AUV navigation ability.

DPCA as aiding sensor without full integration gives slight blurring, but is substantially better than INS only. Assuming linear trajectory or using pure DPCA navigation result in clearly defocused images (as expected). Note that the synthetic aperture is approximately 6 times longer than the CRLB of DPCA micronavigation alone [3], [7]. In addition, this comparison is somewhat unfair to DPCA micronavigation alone since there is a known bias in the DPCA yaw estimate due to imperfect hardware.

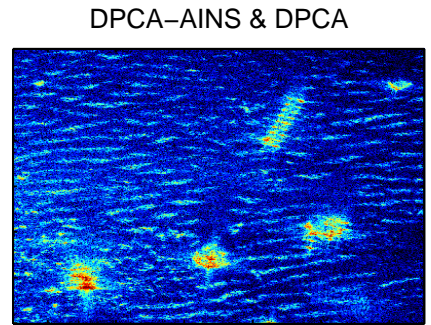

INS \& DPCA

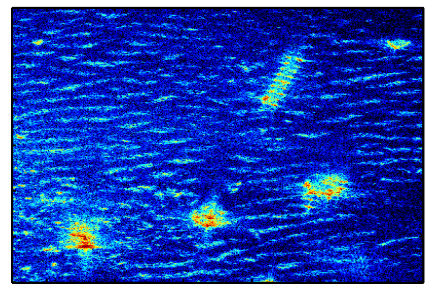

DPCA-AINS
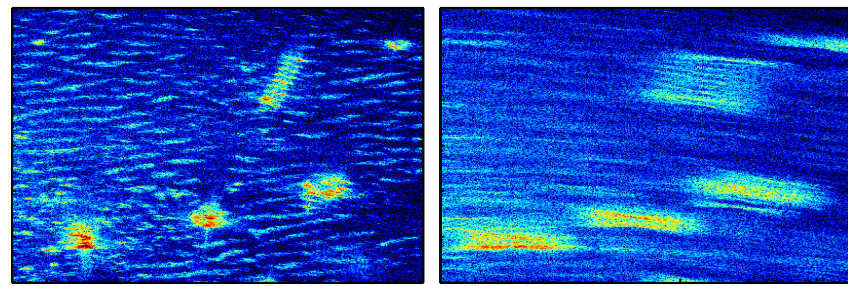

Fig. 9. SAS Images produced by 6 different strategies of combining DPCA and INS.

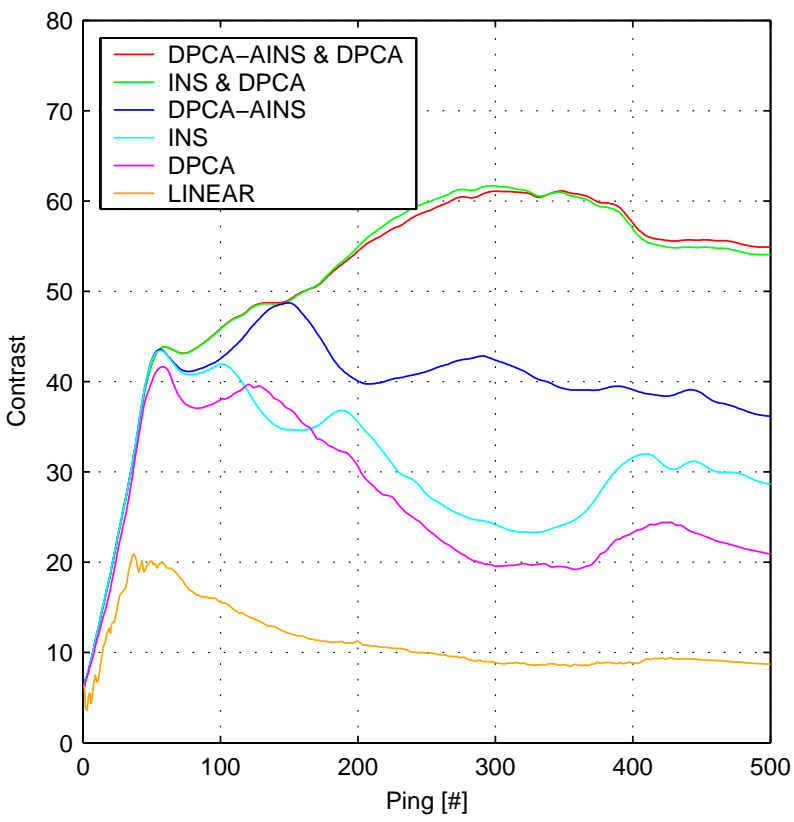

Fig. 10. Cumulative contrast for the corresponding images shown in Fig 9. 


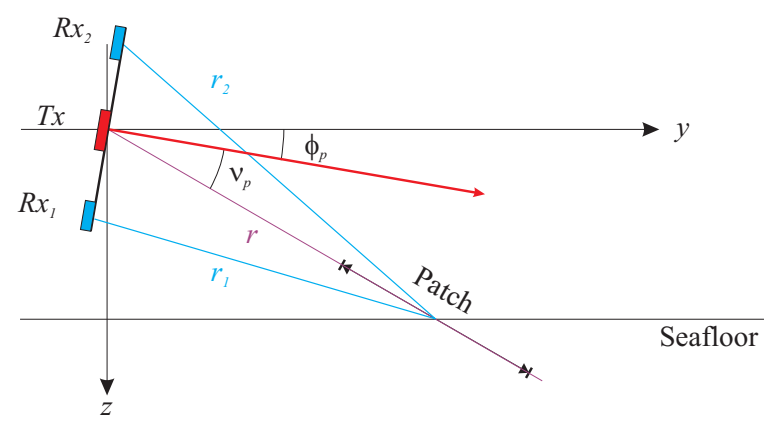

Fig. 11. Principle of interferometric height estimation.

Figure 10 shows the cumulative contrast for the images in Fig 9. All methods except linear assumption are almost equally good after 50 pings $(1.7 \mathrm{~m}$ synthetic aperture). After that, the contrasts diverge as expected. Theoretically, using DPCA as aiding sensor alone should have been sufficient assuming no fluctuations in the ocean. The poor performance may in addition be caused by either an incorrect lever-arm between the IMU and the sonar, or an inaccurate specification of DPCAaccuracy in the Kalman filter. Figure 10 also indicates that full integration of DPCA in addition to DPCA as an aiding-sensor is the optimal method for this dataset.

\section{SWATH BATHYMETRY}

The basic principle of interferometry is to measure the difference in travel time between the returned echos for two separate receiver banks [23]. Figure 11 shows a simplified interferometric geometry for a sonar with roll $\phi_{\mathrm{p}}$. The slantrange direction to the seafloor relative to the platform roll, $\nu_{\mathrm{p}}$, is simply

$$
\nu_{\mathrm{p}}=\arcsin \left(\frac{c \tau}{B}\right),
$$

where $c$ is the sound velocity, $\tau$ the difference in travel time and $B$ the baseline between the receiver banks. The corresponding height above the seafloor, $H$, is then

$$
H=r \sin \left(\nu_{\mathrm{p}}+\phi_{\mathrm{p}}\right),
$$

where $r$ is the distance between the sonar and the seafloor.

Although the principle is the same, a slightly more complicated algorithm is needed when the SAS images from bank 1 and 2 are focused at a specific height.

The non-trivial part of interferometry is, however, estimating the lag accurately. We have tested two different methods. The most robust of these estimates a coarse bathymetric map, based on complex cross correlation of patches along y [20]. For each of the two banks, we select a small stripe of the image along $y$, cross correlate them and estimate the lag. To ensure both sufficient data in the correlation and a useful resolution in the bathymetric maps, we oversample the images along $\mathrm{y}$. Significant overlap is also used in the correlation windows. The resulting bathymetric maps have full resolution in $\mathrm{x}$, but are lowpass filtered in $\mathrm{y}$. This technique is limited by baseline decorrelation (or geometrical distortions) at near range and low Signal to Noise Ratio (SNR) at far range.

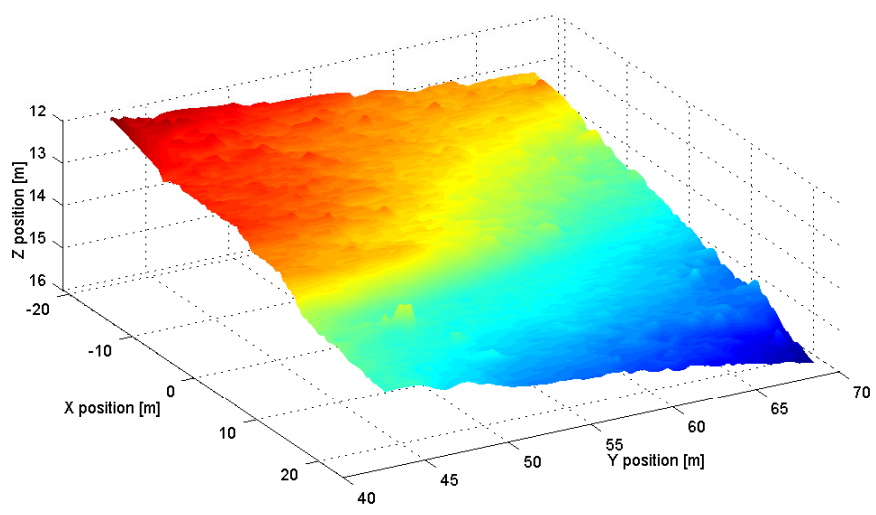

Fig. 12. Bathymetric image of full scene of InSAS-2000.

The bathymetry of the full scene in InSAS-2000 is shown in Fig 12. The rock can be seen at $x=13 \mathrm{~m}, y=48 \mathrm{~m}$. Note that the height deviation is less than $4 \mathrm{~m}$ on an area of approximately $1350 \mathrm{~m}^{2}$. The corresponding SAS-images where calculated with a resolution of $5 \times 0.1 \mathrm{~cm}$ and focused at a flat seafloor at $12.5 \mathrm{~m}$ depth. Then a correlation window of $1 \mathrm{~m}$ is displaced with intervals of $5 \mathrm{~cm}$ along each stripe. The gridding in the bathymetric map is therefore $5 \times 5 \mathrm{~cm}$. Areas with low coherence are interpolated and the resulting data is also smoothed with a block size of $5 \times 5$ pixels. Note that the interpolation fills the shadows in the SAS image with valid heights from nearby areas. This is extremely hazardous, since man-made objects (i.e. mines) within the shadow areas do vanish in the bathymetric map. The proper way to handle this is to leave the shadow areas with no valid height.

Figure 13 shows the three foremost objects from run 221113 (see Fig 5). The rock (leftmost object) is, as expected, most dominant. Compared to the SAS image, there is a small displacement of the objects, which partially is caused by focusing at incorrect height. The shape of the objects are also slightly distorted. The bathymetric map in Fig 13 is calculated with the same parameters as the map in Fig 12, except that the SAS images originally had $1 \mathrm{~cm}$ resolution along-track. Complex averaging is used to resample the images to $5 \mathrm{~cm}$ and consequently the noise is reduced.

Another possible approach is to estimate the lag by computing the interferogram [11]. This method has no smoothing along $\mathrm{y}$ and has potential to achieve the same resolution in the bathymetric map as in the SAS images. However, one of the main difficulties is the need for 2D phase unwrapping. This problem can be avoided by using coarse cross correlation based height as input to the imaging, thus avoiding phase wrapping in the interferogram. Alternatively, the coarse estimate can be used to calculate a synthetic interferogram which is subtracted from the measured interferogram. The calculated height is then a correction to the coarse estimate.

\section{A. Bathymetry in Object Classification}

High resolution bathymetric imagery of objects have potential to dramatically increase the classification ability. The two 


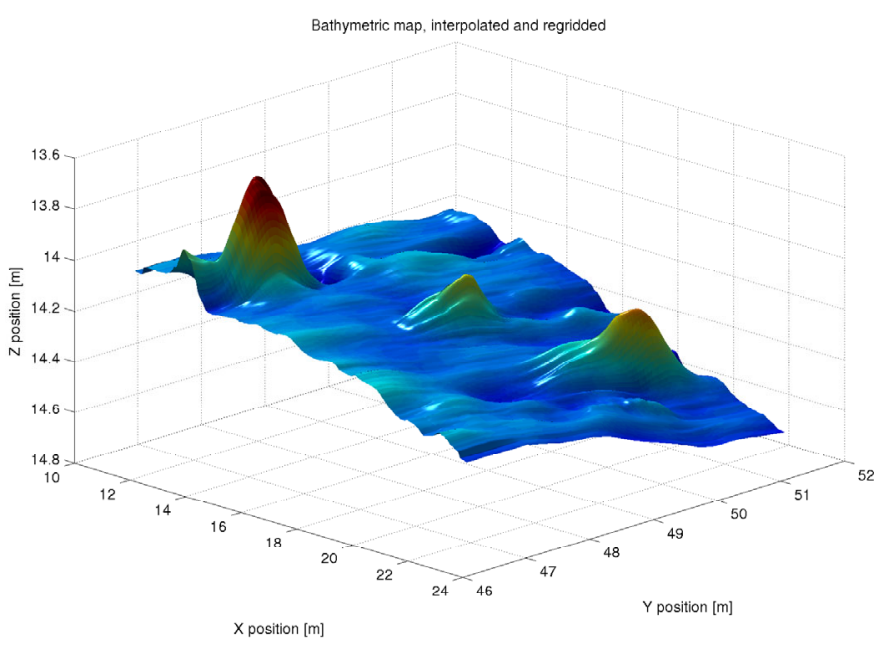

Fig. 13. Bathymetric image of three objects.

techniques outlined above is applied to high resolution images of the rock from run 221107 and run 221113 (see Fig 14). The rock is rotated approximately 90 degrees between the two runs. Figure 15 shows the SAS image (left), coarse bathymetry (center) and full-resolution (right) bathymetry for both runs. The synthetic resolution in the SAS images is originally $1 \times 1$ $\mathrm{mm}$, then resampled to $5 \times 0.1 \mathrm{~cm}$ in the coarse bathymetry and $5 \times 5 \mathrm{~cm}$ in the full resolution method.

In run 221113, the orientation of the rock is visible with both methods, but some additional aspects may be worth commenting: The full resolution bathymetry seems equally smooth as the coarse estimate and also more inaccurate. This is caused by a high noise level in the interferogram. To achieve useful results, we apply median filters, remove bad data and apply smoothing. Therefore, the final map is comparable to the coarse estimate in resolution. The main features are, however, clearly visible in both figures. The rock is oriented along $\mathrm{x}$ with the highest point towards negative $\mathrm{x}$ (see Fig 14).

The rock is rotated approximately 90 degrees in run 221107 (lower part) compared to run 221113 (upper part). The shape of the rock is a bit more distorted in this case, but this is to some extent caused by shadow from the front top. The highest point of the rock is now orientated towards the sonar. In the SAS image we see that the reflectivity at the center of the rock is lower than at the front and back. Hence the high uncertainty

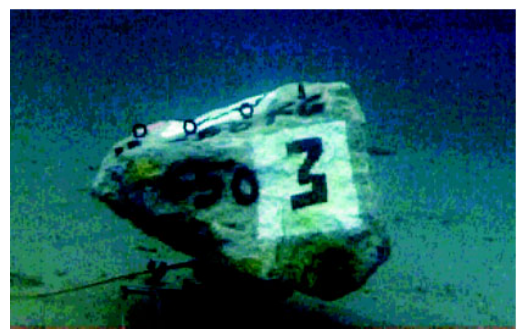

Fig. 14. Optical image of the rock at the turntable. in the bathymetric map at this point. This is consistent with the shape of the rock (see Fig 14). The full resolution bathymetry shows the same features, which are dominated by a large peak at the front and a somewhat lower peak at the back, but in this case the quality of the bathymetric map is lower.

A major limitation to both techniques of bathymetric processing is grating lobes caused by imperfect navigation, and sidelobes. Both effects reduce the ability to properly map areas around strong reflectors, something that is essential for proper 3D object shaping with bathymetry.

\section{Conclusion}

The challenge in seafloor imaging in military applications, is to produce images of such quality that man-made objects (e.g. mines) can be separated from natural objects (rocks) of equal size with highest possible presision. To maintain high area coverage rate in cluttered environments, the sonar image resolution at far range (detection ranges) must be of "classification quality". SAS seems to be an excellent tool for this.

A limiting factor for practical use of SAS has been the requirement for navigation accuracy. This problem is solved by fusion of sonar micronavigation with the aided INS onboard the AUV. This requires knowledge of the slant-range direction, which can only be obtained by estimating the swath bathymetry with an interferometric sensor (or prior knowledge of the seafloor). This has been solved by assuming flat seafloor for non-interferometric sensors.

Operating AUVs in areas with rough topography is optimally performed by running at constant height. This leads to out-of-plane motion deviations, which again causes defocusing without prior knowledge of the bathymetry [12]. This effect is independent of choice of beamforming technique. There are techniques to compensate for this which are valid under certain assumptions [13]. However, the only general solution for this problem is either running on a straight line (obtaining a focused but not correctly positioned image), or estimating the height by the use of interferometry.

Full swath cross-correlation based SAS interferometry seems feasible, and has the potential to dramatically increase the along-track resolution compared to traditional bathymetric mapping with Multi Beam Echosounders (MBE). Interferometric SAS does however have the same limitation as MBEs to height accuracy, given by the requirement of known sound velocity profile. A two-sided interferometric SAS in combination with a MBE as gap-filler seems as the natural choice for Rapid Environmental Assesment (REA) type AUV operations, where the requirement for absolute height accuracy is relaxed. High resolution mapping of objects will increase classification ability. This is, however, non-trivial, and considerable work is left before reliable 3D mapping of objects can be done.

\section{ACKNOWLEDGMENT}

The authors would like to thank the SACLANTCEN team and the QinetiQ team involved in the InSAS-2000 trials. We especially thank Dr Marc Pinto and Andrea Bellettini at SACLANTCEN for many fruitful discussions. 

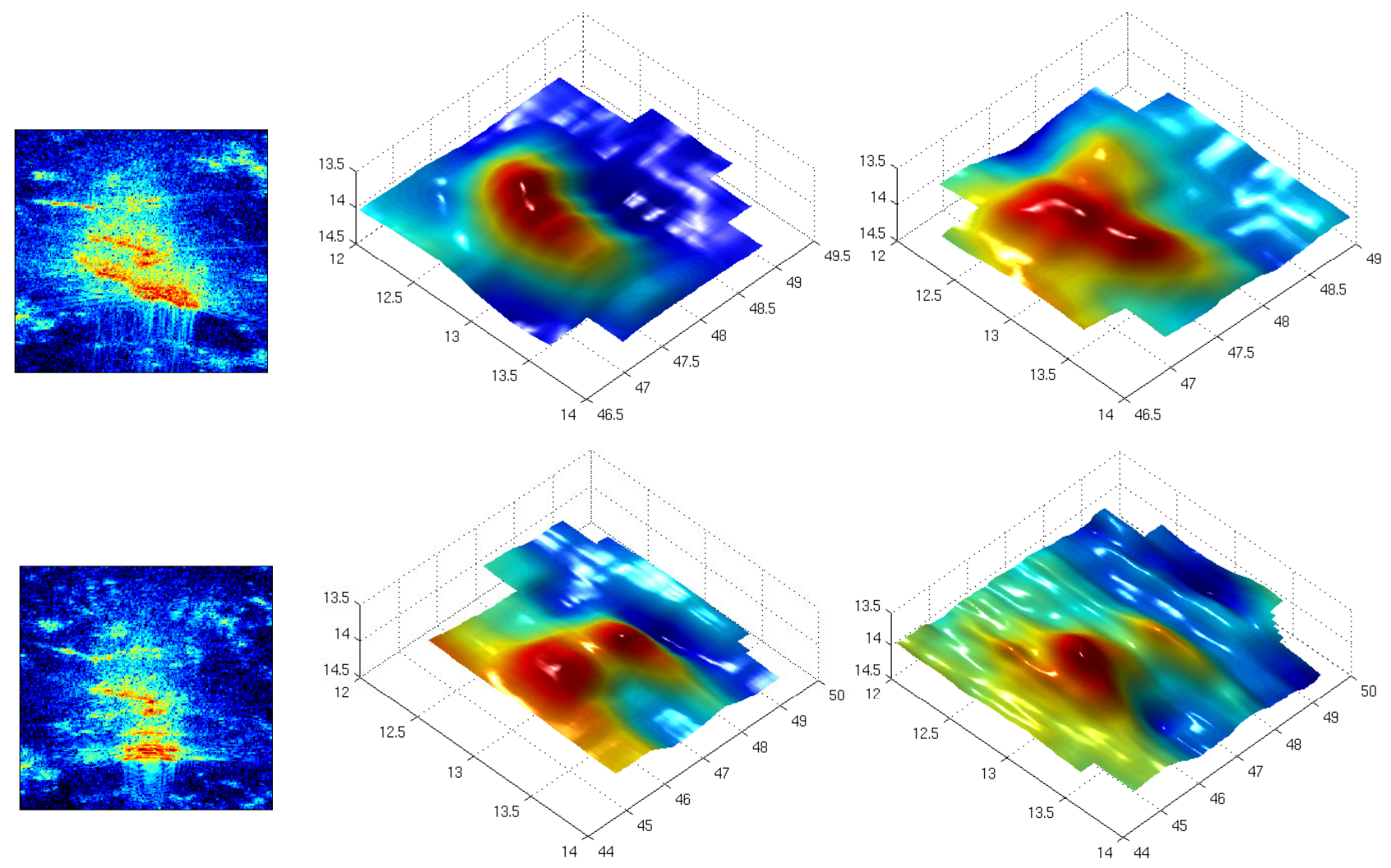

Fig. 15. Upper row: SAS image (left), coarse bathymetry (center) and phase unwrapped bathymetry (right) of run 221113. Lower row: SAS image (left), coarse bathymetry (center) and phase unwrapped bathymetry (right) of run 221107 . The rock is rotated $9 \odot$ between the upper and lower images.

\section{REFERENCES}

[1] P. E. Hagen, R. E. Hansen, K. Gade, and E. Hammerstad, "Interferometric synthetic aperture sonar for AUV based mine hunting: The SENSOTEK project," in Proceedings of Unmanned Systems 2001, Baltimore, MD, USA, July-August 2001.

[2] P. E. Hagen, N. Størkersen, K. Vestgård, and P. Kartvedt, "The HUGIN 1000 autonomous underwater vehicle for military applications," in Proceedings of Oceans 2003 MTS/IEEE, San Diego, CA, USA, September 2003, pp. 1141-1145.

[3] L. Wang, A. Bellettini, R. Hollett, A. Tesei, M. Pinto, S. Chapman, and K. Gade, "InSAS'00: Interferometric SAS and INS aided SAS imaging," in Proceedings of Oceans 2001 MTS/IEEE, Honolulu, HI, USA, November 2001, pp. 179-187.

[4] M. A. Pinto, S. Fioravanti, and E. Bovio, "Multiple-element synthetic aperture sonar design," Saclantcen note, 1998.

[5] _ - "Accuracy of synthetic aperture sonar using a displaced phase center antenna," Saclantcen Memorandum SM-352, 1998.

[6] D. Billon and F. Fohanno, "Theoretical performance and experimental results for synthetic aperture sonar self-calibration," in Proceedings of Oceans 98, Nice, France, September 1998, pp. 965-970.

[7] A. Bellettini and M. Pinto, "Theoretical accuracy of synthetic aperture sonar micronavigation using a displaced phase centre antenna," IEEE J. Oceanic Eng., vol. 27 (4), pp. 780-789, 2002.

[8] B. Jalving, K. Gade, O. K. Hagen, and K. Vestgård, "A toolbox of aiding techniques for the HUGIN AUV integrated inertial navigation system," in Proceedings of Oceans 2003 MTS/IEEE, San Diego, CA, USA, September 2003, pp. 1146-1153.

[9] M. Soumekh, Fourier Array Imaging. Prentice Hall, 1994.

[10] D. W. Hawkins, "Synthetic aperture imaging algorithms: with application to wideband sonar," Ph.D. dissertation, University of Canterbury, Christchurch, New Zealand, 1996.

[11] G. Franceschetti and R. Lanari, Synthetic Aperture Radar Processing. CRC Press, 1999
[12] J. C. V. Jakowatz, D. E. Wahl, D. C. Ghiglia, and P. A. Thompson, Spotlight-Mode Synthetic Aperture Radar: A Signal Processing Approach. Kluwer Academic Publishers, 1996.

[13] H. Groen, R. E. Hansen, and J. C. Sabel, "Sonar path correction in synthetic aperture processing," in Proceedings of UDT Europe 2003, Malmö, Sweden, June 2003.

[14] D. H. Johnson and D. E. Dudgeon, Array Signal Processing: Concepts and Techniques. Prentice Hall, 1993.

[15] L. M. H. Ulander, H. Hellsten, and G. Stenström, "Synthetic-aperture radar processing using fast factorised back-projection," submitted to IEEE Trans. Aerospace and Electronic Systems, 2001.

[16] G. Shippey, J. Pihl, and M. Jönsson, "Autopositioning for synthetic aperture sonar using fast factorised back projection," in Proceedings of CAD/CAC 2001, Halifax, Canada, November 2001.

[17] S. Banks, "Studies in high resolution synthetic aperture sonar," $\mathrm{Ph} . \mathrm{D}$ dissertation, University College London, London, UK, 2002.

[18] K. Warman, K. Chick, and E. Chang, "Synthetic aperture sonar processing for widebeam/broadband data," in Proceedings of Oceans 2001 MTS/IEEE, Honolulu, HI, USA, November 2001, pp. 208-211.

[19] E. Chang, "Synthetic aperture sonar - tutorial part II," Presented at Oceans 2001 MTS/IEEE, Honolulu, HI, USA, November 2001.

[20] M. Pinto, R. D. Hollett, A. Bellettini, and S. Chapman, "Bathymetric imaging with wideband interferometric synthetic aperture sonar," submitted to IEEE J. Oceanic Eng., 2001.

[21] D. Billon and F. Fohanno, "Two improved ping-to-ping cross correlation techniques for synthetic aperture sonar: theory and sea results," in Proceedings of Oceans 2002 MTS/IEEE, Biloxi, MS, USA, October 2002, pp. 2284-2293.

[22] W. W. Bonifant Jr, M. A. Richards, and J. H. McClellan, "Interferometric height estimation of the seafloor via synthetic aperture sonar in the presence of motion errors," IEE Proc. Radar, Sonar Navig., vol. 147 (6), pp. 322-330, 2001.

[23] X. Lurton, "Swath bathymetry using phase difference: Theoretical analysis of acoustical measurement precision," IEEE J. Oceanic Eng., vol. 25 (3), pp. 351-363, 2000 . 